American Journal of Immunology 6 (1): 11-14, 2010

ISSN 1553-619X

(C) 2010 Science Publications

\title{
Anti-Cardiolipin Antibody in Acute Myocardial Infarction
}

\author{
${ }^{1}$ Abdolreza Sotoodeh Jahromi, ${ }^{2}$ Mohammad Shojaie, \\ ${ }^{3}$ Samira Dana and ${ }^{4}$ Abdoulhossain Madani \\ ${ }^{1}$ Department of Immunology, \\ ${ }^{2}$ Department of Cardiology, \\ ${ }^{3}$ Department of Student Research Committee, \\ Jahrom University of Medical Science, Jahrom, Iran \\ ${ }^{4}$ Department of Public Health, Hormozgan University of Medical Science, \\ Bandar Abbas, Iran
}

\begin{abstract}
Problem statement: Myocardial infarction is the combined result of environmental and personal factors. Data concerning the relation between anti-Phospholipid (aPL) antibodies and myocardial infarction in subjects without evidence of overt autoimmune disease are conflicting. Anticardiolipin antibody is detected in various diseases like rheumatoid arthritis, systemic lupus erythematosus and anti-phospholipid antibody syndrome. The study of Anticardiolipin antibody in Acute Myocardial Infarction (AMI) might shed light on etiologic mechanisms in the pathogenesis of acute coronary syndromes. The purpose of the present study was to determine association of plasma aPL antibodies, namely, anti-Cardiolipin (aCL) antibodies, with AMI. Approach: This study recruited 45 patients with the diagnosis of AMI according to WHO criteria in their first $24 \mathrm{~h}$ of admission. Thirty six matched individuals were studied as the control group with normal coronary artery angiography. Samples were tested for IgG-class antibodies to cardiolipin by an ELISA and the results were compared. Results: There were not significant differences between plasma level of aCLAs IgG in the patients with AMI on admission ant the control group. Also aCLAs IgG was not correlated with hypertension, diabetes mellitus, hyperlipidemia, sex, age and smoking. Conclusion: Our findings suggest that aCLAs IgG are not indicative of hypercoagulable state in patients with AMI.
\end{abstract}

Key words: Anticardiolipin antibody, myocardial infarction

\section{INTRODUCTION}

Myocardial Infarction (MI) is the combined result of environmental factors and Personal predispositions (Zimmerman et al., 1995). Prothrombotic factors may play a more important role in these patients. Various prothrombotic factors and markers of endothelial damage have been associated with an increased risk of myocardial infarction e.g., fibrinogen (Thompson et al., 1995).

In addition to mentioned factors autoantibodies may play a more important role in these patients. Various autoantibodies have been associated with an increased risk of myocardial infarction e.g., antiphosphospholipid antibodies (Hughes, 2010).

Antibodies binding to anionic phospholipids, such as cardiolipin, are associated with a clinical syndrome characterized in particular by venous and arterial thrombosis, recurrent abortion and thrombocytopenia. Although the anti-Phospholipid (aPL) antibody syndrome was first described in patients with Systemic Lupus Erythematosus (SLE), it is now generally accepted that there is a group of patients in whom high titers of aPL antibodies, usually IgG class and thrombotic features occur without clinical manifestations of SLE. On the other hand, aPL antibodies may transiently appear during many infections and in association with several drugs without any association with thrombosis (McNeil et al., 1991).

The investigation of anti-Cardiolipin Antibodies (aCLAs) in MI might shed light on hypercoagulability mechanisms in the pathogenesis of acute coronary syndromes. There were a few documents about aPL syndrome in Iranian patients with AMI.

Our goal was to study the plasma level of aCLAs in our patients who developed acute myocardial infarction and healthy control group and to analyze their relationship with traditional cardiovascular risk factors.

Corresponding Author: Mohammad Shojaie, Department of Cardiology, Jahrom University of Medical Sciences, Jahrom, Iran 


\section{MATERIALS AND METHODS}

Subjects: This case-control study recruited 45 consecutive patients with Acute Myocardial Infarction (AMI) including 31 men and 14 women with the mean age of $62.7 \pm 13.1$ years old who were taken to the emergency room of Peymanieh Hospital of Jahrom, Iran, with the chief complaint of chest pain from Feb. 2007 to May 2008.

We also selected 36 individuals that referred to the emergency room with chest pain with normal coronary angiography as our control group and matched them for age, sex and other CAD risk factors such as Hypertension (HTN), Diabetes Mellitus (DM) and Hyperlipidemia (HLP).

The study protocol was approved by research ethics committee of Jahrom University of Medical Sciences and informed consents were obtained from all participants before enrollment. A questionnaire including information about the past medical and drug history (HTN, HLP, DM, smoking, chronic diseases such as collagen vascular diseases and asthma), family history of Coronary Artery Disease (CAD) and demographic information was completed for each patient.

The exclusion criteria were the presence of severe liver disease, malignancy, recent cardiac surgery, angioplasty, stable or unstable angina, receiving of anticoagulant drugs, hemolysis, pregnancy loss, history of deep vein or arterial thrombosis, inflammatory and rheumatologic diseases such as collagen vascular disease, SLE and APA syndrome.

Definitions: AMI was defined as chest pain lasting more than 30 minutes accompanied by ischemic electrocardiographic changes and was confirmed by the presence of total Creatinine Phosphokinase (CPK) or MB fraction levels of more than twice the upper normal limit (Antman et al., 2008). The absence of any narrowing in coronary artery diameter was considered as normal coronary angiography.

Blood pressure was measured two times in sitting position after $5 \mathrm{~min}$ of rest using a mercury sphygmomanometer. Hypertension was defined as blood pressure more than $130 / 85 \mathrm{mmHg}$ or use of any antihypertensive medication (Virella and Lopes-Virella, 2008). DM was defined by a physician's diagnosis, a fasting plasma glucose level of $\geq 126 \mathrm{mg} \mathrm{dL}^{-1}$ or use of diabetic medications (Foster and Pitts, 2009). Echocardiography was done for all patients during their hospital stay by one cardiologist. Ejection Fraction (EF) is defined to be normal $(>55 \%)$, mild $(45-54 \%)$, moderate $(30-44 \%)$ and $(>30 \%)$ severe LV systolic dysfunction (Lang et al., 2005).
Laboratory analysis: Fasting levels of plasma total cholesterol, High Density Lipoprotein (HDL) cholesterol, Low Density Lipoprotein Cholesterol (LDL) and Triglycerides (TG) were measured in Research Laboratory of Jahrom Medical University. Total cholesterol and Triglyceride levels were measured by enzymatic techniques using a Selectra E biochromatic analyzer. HDL and LDL cholesterol level was measured after glucose levels were measured by the glucose oxidase method. CPK were measured by an enzymatic method.

Blood samples $(5 \mathrm{cc})$ were obtained by venipuncture from the patients immediately after admission before starting any IV medications by trained staff and for lipid profile and fasting blood sugar at the first $24 \mathrm{~h}$ of AMI after $12 \mathrm{~h}$ of fasting. In control subjects all blood sample were obtained after $12 \mathrm{~h}$ of fasting then plasma was separated and frozen at $-70^{\circ} \mathrm{C}$ for later processing. Level of circulating anticardiolipin IgG antibodies were determined by Enzyme Linked Immunosorbent Assay (ELISA) Aeskulisa, REF: 7204, Germany according to manufacturer's recommendations. We consider aCLAs level above $15 \mathrm{ng} \mathrm{mL}^{-1}$ and aANVAs level above1.18 $\mathrm{ng} \mathrm{mL}^{-1}$ as positive results.

Statistical analysis: Statistical analysis was performed by SPSS (version 15; SPSS, Inc., Chicago, IL). Data were expressed as mean $\pm 1 \mathrm{SD}$. Continuous variables with little-to-mild skewness were summarized as mean $\pm \mathrm{SD}$ and compared using Student's t-test. Discrete variables were presented as frequencies and group percentages. Nominal variables were tested with Pearson's 2 test and Binary variables were tested with the chi square test. Generalized Linear Models were used to adjust smoking between two groups. All tests were two-tailed with a 0.05 type I error rate. ANOVA and Kruskal-Wallis test were used to evaluate association of aCLAs with different type of AMI and EF.

\section{RESULTS}

The demographic and clinical characteristics as well as laboratory variables of the study groups are shown in Table 1. In patient group 6 cases (14\%) had Non-ST Elevation MI (NSTEMI) and 37 (86\%) had ST Elevation MI (STEMI). There were no significant differences between the two groups regarding the following variables: age, sex, HTN, DM, LDL, HDL, total cholesterol and TG. Also no significant differences were not found between plasma levels of aCLAs IgG in the patient group ant the control group (Table 1). 
Am. J. Immunol., 6 (1): 11-14, 2010

Table 1: Demographic and clinical characteristics of the study groups

\begin{tabular}{|c|c|c|c|}
\hline Variable & $\begin{array}{l}\text { Case group } \\
\mathrm{n}=45\end{array}$ & $\begin{array}{l}\text { Control group } \\
\mathrm{n}=36\end{array}$ & p-value \\
\hline Age, (years) & $62.7 \pm 13.1$ & $60.1 \pm 11.9$ & 0.38 \\
\hline Male, n (\%) & $31(68.9 \%)$ & $18(50 \%)$ & 0.08 \\
\hline Current smoker, n (\%) & $11(24.4 \%)$ & $2(5.6 \%)$ & $0.02 *$ \\
\hline HTN, n (\%) & $8(17.8 \%)$ & $11(30.6 \%)$ & 0.18 \\
\hline Type 1 DM, n (\%) & $2(4.4 \%)$ & $4(11.1 \%)$ & 0.40 \\
\hline Type 2 DM, n (\%) & $7(5.6 \%)$ & $4(11.1 \%)$ & 0.56 \\
\hline Total Cholesterol $\left(\mathrm{mg} \mathrm{dL}^{-1}\right)$ & $189.2 \pm 43.7$ & $176.3 \pm 32.3$ & 0.14 \\
\hline LDL-C $\left(\mathrm{mgdL}^{-1}\right)$ & $112.8 \pm 35.6$ & $106 \pm 28.7$ & 0.36 \\
\hline HDL-C $\left(\mathrm{mg} \mathrm{dL}^{-1}\right)$ & $46.2 \pm 11.4$ & $42.3 \pm 10$ & 0.11 \\
\hline LDL/HDL ratio & 4.23 & 4.24 & 0.96 \\
\hline Triglyceride $\left(\mathrm{mg} \mathrm{dL}^{-1}\right)$ & $146.1 \pm 97.6$ & $153 \pm 105.5$ & 0.76 \\
\hline aCLAs (ng mL ${ }^{-1}$ ) & $21.7 \pm 55.7$ & $13.9 \pm 38.1$ & 0.46 \\
\hline Positive aCLAs, n (\%) & $8(18.6 \%)$ & $5(13.2 \%)$ & 0.53 \\
\hline
\end{tabular}

We examined the association between plasma aCLAs IgG and selected cardiovascular risk factors. There was not a significant correlation between aCLAs IgG in patients and controls groups. Also, we didn't find a significant association between plasma aCLAs IgG with HTN, Type 1 DM, Type 2 DM, age, sex, LDL, HDL, TG, total cholesterol and adjusted smoking. We didn't find a statically significant association of plasma aCLAs IgG with type of MI, LV systolic Function (EF) and mortality in our cases and with sex and not with age in all subjects.

\section{DISCUSSION}

In the present study, no significant differences were not found between the aCL antibody levels in the patients and the control groups.

Thus, the results of our prospective study are in agreement with three earlier researches, (Gaeta et al., 1998; Phadke et al., 1993; Rebic et al., 1993), but they disagree with findings of three other studies of the prevalence of aCL antibodies in patients with myocardial infarction (Yilmaz and Yilmaz, 1994; Ferlazzo et al., 1993).

There may be several reasons for these discrepancies. First, there are differences in the study populations. Second,_differences in test techniques may be involved. There is evidence that aPL antibodies in patients with SLE are directed against an antigenic complex containing not only anionic phospholipids but also a plasma apolipoprotein $\mathrm{H}$ ( $\beta_{2}$-glycoprotein I) (McNeil et al., 1990). On the other hand, aCL antibodies occurring in infectious diseases appear to bind to pure anionic phospholipids (Hunt et al., 1992).

Also, there is accumulating evidence to suggest that infections can play a role in the pathogenesis of coronary heart disease (Lopes-Virella et al., 2008). A transient aCL antibody response takes place in a variety of bacterial and viral infections and elevated levels can persist in many chronic infections, notably syphilis (McNeil et al., 1991). Thus, it is possible that aCL antibodies in our study subjects reflect some chronic infection, for example, chlamydial infection, which has been associated with coronary heart disease (Saikku et al., 1988; 1992; Thom et al., 1991). Actually, antibodies against chlamydial lipopolysaccharide may cross-react with anionic phospholipids in ELISA (Vaarala, 1991). We have not excluded infectious cases in two our groups of study.

In accordance with this, Gaeta et al. (1998); Vaarala et al. (1995); Phadke et al. (1993) and Rebic et al. (1993) reported no association between elevated levels of aCL antibodies and acute myocardial infarction.

\section{CONCLUSION}

This study shows no significant association between anticardiolipin antibody concentrations and acute myocardial infarction. However, according to results our study and previous studies, there are controversial on association of ACLA and AMI. The importance of serum anticardiolipin antibody levels in acute myocardial infarction is still undetermined and remains to be clarified.

\section{ACKNOWLEDGEMENT}

This study was completely financed by Student Research Committee (SRC) of Jahrom University of Medical Sciences. The authors are grateful to the patients and the control individuals who accepted to enter this study. This article has been extracted from Ms Dana's thesis.

\section{REFERENCES}

Antman, E.M., M. Hand, P.W. Armstrong, E.R. Bates and L.A. Green et al., 2008. Focused update of the ACC/AHA 2004 Guidelines for the management of patients with ST-elevation myocardial infarction: A report of the American College of Cardiology/American Heart Association Task Force on Practice Guidelines: Developed in Collaboration With the Canadian Cardiovascular Society endorsed by the American Academy of Family Physicians: 2007 Writing Group to Review New Evidence and Update the ACC/AHA 2004 Guidelines for the Management of Patients With ST-Elevation Myocardial Infarction, Writing on Behalf of the 2004 Writing Committee. Circulation, 117: 296-329. PMID: 18071078 
Ferlazzo, B., D. Bonanno, P. Quattrocchi, A.M. Paino and F. Arrigo, 1993. Connection between ischemic heart disease and anticardiolipin antibodies positivity. Minerva Cardioangiol, 41: 113-117. PMID: 8332265

Foster, J.J. and W. Pitts, 2009. Implementation of an insulin therapy protocol: Applying the Baldrige approach. Am. J. Health Syst. Pharm., 66: 1035-1038. PMID: 19451615

Gaeta, G., S. Lupoli, V. Brancaccio, L. Effuso and V. Russo et al., 1998. Anticardiolipin antibodies and early infarct of the myocardium. Cardiologia, 43: 731-5. PMID: 9738331

Hughes, G.R., 2010. Antiphospholipid syndrome (Hughes syndrome): 10 clinical topics. Lupus, 19: 343-346. PMID: 20353967

Hunt, J.E., H.P. McNeil, G.J. Morgan, R.M. Crameri and S.A. Krilis, 1992. A phospholipid beta 2glycoprotein I complex is an antigen for anticardiolipin antibodies occurring in autoimmune disease but not with infection. Lupus, 1: 75-81. PMID: 1301967

Lang, R.M., M. Bierig, R.B. Devereux F.A. Flachskampf and A. Foster et al., 2005. Quantification writing group; American society of echocardiography's guidelines and standards committee; European association of echocardiography: Recommendations for chamber quantification: A report from the American society of echocardiography's guidelines and standards committee and the chamber quantification writing group, developed in conjunction with the European association of echocardiography, a branch of the European society of cardiology. J. Am. Soc. Echocardiogr., 18: 1440-14463. PMID: 16376782

Lopes-Virella, M.F., R.E. Carter, G.E. Gilbert, R.L. Klein and M. Jaffa et al., 2008. Diabetes Control and Complications Trial/Epidemiology of Diabetes Intervention and Complications Cohort Study Group. Risk factors related to inflammation and endothelial dysfunction in the DCCT/EDIC cohort and their relationship with nephropathy and macrovascular complications. Diabetes Care, 31: 2006-2012. PMID: 18628568

McNeil, H.P., C.N. Chesterman and S.A. Krilis, 1991. Immunology and clinical importance of antiphospholipid antibodies. Adv. Immunol., 49: 193-280. PMID: 1853785

McNeil, H.P., J.E. Hunt and S.A. Krilis, 1990. New aspects of anticardiolipin antibodies. Clin. Exp. Rheumatol., 8: 525-527. PMID: 2127002

Phadke, K.V., R.A. Phillips, D.T. Clarke, M. Jones and P. Naish et al., 1993. Anticardiolipin antibodies in ischaemic heart disease: Marker or myth? Br. Heart J., 69: 391-4. PMID: 8518060
Rebic, R., D. Nastic-Miric, S. Pavlovic, Z. Pokrajcic and V. Bosnjakovic, 1993. Measuring of cardiolipin antibodies and plasma lipids in different stages of coronary disease. Med. Pregl. Suppl., 1: 20-22. PMID: 8569595

Saikku, P., M. Leinonen, K. Mattila, M.R. Ekman and M.S. Nieminen et al., 1988. Serological evidence of an association of a novel chlamydia, TWAR, with chronic coronary heart disease and acute myocardial infarction. Lancet, 2: 983-986. PMID: 2902492

Saikku, P., M. Leinonen, L. Tenkanen, E. Linnanmaki and M.R. Ekman et al., 1992. Chronic chlamydia pneumoniae infection as a risk factor for coronary heart disease in the Helsinki heart study. Ann. Intern. Med., 116: 273-278. PMID: 1733381

Thom, D.H., S.P. Wang, J.T. Grayston, D.S. Siscovick and D.K. Stewart et al., 1991. Chlamydia pneumoniae strain TWAR antibody and angiographically demonstrated coronary artery disease. Arterioscler. Thromb., 11: 547-551. PMID: 2029495

Thompson, S.G., J. Kienast, S.D.M. Pyke, F. Haverkate and J.C.W. Van de Loo, 1995. Hemostatic factors and the risk of myocardial infarction or sudden death in patients with angina pectoris. N. Engl. J. Med., 332: 635-641. PMID: 7845427

Vaarala, O., 1991. Binding profiles of anticardiolipin antibodies in sera from patients with SLE and infectious diseases. J. Autoimmun., 4: 819-830. PMID: 1797029

Vaarala, O., M. Mänttäri, V. Manninen, L. Tenkanen, and M. Puurunen et al., 1995. Anti-cardiolipin antibodies and risk of myocardial infarction in a prospective cohort of middle-aged men. Circulation, 91: 23-27. PMID: 7805207

Virella, G. and M.F. Lopes-Virella, 2008. Atherogenesis and the humoral immune response to modified lipoproteins. Atherosclerosis, 200: 239-246. PMID: 18513726

Yilmaz, A.K. and C. Yilmaz, 1994. Importance of serum anticardiolipin antibody levels of coronary heart disease. Clin. Cardiol., 17: 117-121. PMID: 8168279

Zimmerman, F.H., A. Cameron and L.D. Fisher, 1995. Myocardial infarction in young adults: angiographic characterization, risk factors and prognosis (coronary artery surgery study registry). J. Am. Coll. Cardiol., 26: 654-661. PMID: 7642855 